\title{
Numerical Solution of MRLW Equation with İmplicit Finite- Difference Approximation
}

\author{
Melike Karta \\ Department of Mathematics, Faculty of Science and Arts, \\ Agri Ibrahim Cecen University, Agri-Turkey \\ E-mail: mkarta@agri.edu.tr
}

\begin{abstract}
In this paper, a numerical solution of the modified regularized long wave (MRLW) equation has been showed using the classical implicit finite-difference. Error norms $L_{2}$ and $L_{\infty}$ have been calculated to show performance of present method. Calculated values are compared with study available in the literature.
\end{abstract}

Keywords: Classical implicit finite-difference technique, MRLW equation.

DOI: $10.7176 / \mathrm{JSTR} / 5-3-09$

\section{Introduction}

The modified regularized long wave (MRLW) equation is related to MEW equation, mKDW equation and RLW equation respectively; MEW equation

mKDW equation

$$
U_{t}+\epsilon U^{2} U_{x}-\mu U_{x x t}=0
$$

and RLW equation

$$
U_{t}+\in 6 U^{2} U_{x}+\mu U_{x x x}=0
$$

$$
U_{t}+U_{x}+\delta U U_{x}-\mu U_{x x t}=0
$$

where $\delta, \epsilon$ and $\mu$ are positive parameters. (MRLW) equation has been stuied by many author. The equation have been introduced a mathematical theory of the equation by Benjamin et al. Bona and Pryant have proposed the existence and uniqueness of the equation. Esen and Kutluay, Gardner and Gardner have finite element method for solution of the MRLW equation. Gou and Cao have used pseudo-spectral method for he solution of the MRLW equation. Karakoç et al. obtained a numerical solution of the modified regularized long wave (MRLW) equation using a numerical technique based on lumped Galerkin method using cubic B-spline finite elements. Karakoç et al. applied a method based on collocation of quintic B- spline and Petrov Galerkin finite element method in which the element shape functions are cubic and weight fuctions are quadratic B-spline for numerical solutions of (MRLW) equation.Keskin and Irk proposed two finite difference approximations for the space discretization and a multi-time step method fort the time discretization fort the (MRLW) equation.

In this paper, we applied the classical implicit finite-difference scheme to solve the MRLW equation. We used mathlab program to obtain numerical results.

\section{Application of the Method}

In this paper, we will consider the MRLW equation

$$
U_{t}+U_{x}+6 U^{2} U_{x}-\mu U_{x x t}=0
$$

With physical boundary conditions $U \rightarrow 0$ as $x \rightarrow_{-}^{+} \infty$, where $\mu$ is a positive parameter and $x$ is space step, $t$ is time step.To apply numerical method the MRLW equation, we will take solution domain on interval $a \leq x \leq b$. The modified regularized long wave (MRLW) equation has boundary-initial conditions with following form

$$
\begin{aligned}
& U(a, t)=0, U(b, t)=0 \\
& U(x, 0)=\sqrt{c} \operatorname{sech}\left[p\left(x-x_{0}\right)\right]
\end{aligned}
$$


Where $c=1, p=\sqrt{\frac{c}{\mu(c+1)}}$ and $x_{0}=40$. Exact solution of the MRLW equation

$$
U(x, t)=\sqrt{c} \operatorname{sech}\left[p\left(x-(c+1) t-x_{0}\right)\right]
$$

The interval $[a, b]$ is divided into $N$ equal subinterval such that $a<x_{0}<x_{1}<\ldots<x_{N}=b$ for $m=0,1, \ldots, N$ at the nodal points $x_{m}$ by selecting the space step size as $h=\frac{b-a}{N}=\left(x_{m+1}-x_{m}\right)$.

Using the forward difference approximation for $U_{t}, U_{x x t}$,

$$
U_{t}=\frac{U_{m}^{n+1}-U_{m}^{n}}{\Delta t}
$$

and approximation central difference for $U_{x}$

$$
U_{x}=\frac{U_{m+1}-U_{m-1}}{2 h}
$$

and implicit finite-difference approximation for $U^{2} U_{x}$ in equation (1) lead to

$$
\frac{U_{m}^{n+1}-U_{m}^{n}}{\Delta t}+\frac{1}{2}\left[\frac{U_{m+1}^{n+1}-U_{m-1}^{n+1}}{2 h}\right]+6\left(U_{m}^{n}\right)^{2}\left[\frac{U_{m+1}^{n+1}-U_{m-1}^{n+1}}{2 h}\right]-\frac{\mu}{k}\left[U_{x x}^{n+1}-U_{x x}^{n}\right]=0
$$

and we obtain

$$
\begin{gathered}
{\left[-\frac{1}{2 h}-6 \frac{\left(U_{m}^{n}\right)^{2}}{2 h}-\frac{\mu}{k h^{2}}\right] U_{m-1}^{n+1}+\left[\frac{1}{k}+\frac{2 \mu}{k h^{2}}\right] U_{m}^{n+1}} \\
+\left[\frac{1}{2 h}+\frac{6\left(U_{m}^{n}\right)^{2}}{2 h}-\frac{\mu}{k h^{2}}\right] U_{m+1}^{n+1}=U_{m-1}^{n}\left[-\frac{\mu}{k h^{2}}\right]+\left[\frac{1}{k}+\frac{2 \mu}{k h^{2}}\right] U_{m}^{n}-U_{m+1}^{n}\left[\frac{\mu}{k h^{2}}\right]
\end{gathered}
$$

for $m=1,2, \ldots, N$.

\section{Numerical examples and results}

The modified regularized long wave (MRLW) equation to show the performance of the method, error norms $L_{2}$ and $L_{\infty}$ are calculated

$$
L_{2}=\left\|U^{\text {exact }}-U_{N}\right\|_{2} \cong \sqrt{h \sum_{j=0}^{N}\left|U_{j}^{\text {exact }}-\left(U_{N}\right)_{j}\right|^{2}}
$$

and the error norm $L_{\infty}$

$$
L_{\infty}=\left\|U^{\text {exact }}-U_{N}\right\|_{\infty} \cong \max \left|U^{\text {exact }}-\left(U_{N}\right)_{j}\right|
$$

Table

$\mathrm{tf}=10,0 \leq x \leq 100$

\begin{tabular}{|l|l|l|l|l|}
\hline & \multicolumn{2}{|c|}{ finite diffrence } & \multicolumn{2}{c|}{ Keskin-Irk } \\
\hline $\mathrm{h}, \Delta \mathrm{t}$ & $L_{2}$ & $L_{\infty}$ & $L_{\infty}$ & \\
\hline $\mathrm{h}=05, \Delta \mathrm{t}=0.001$ & 0.4829 & 0.2504 & 0.1364 & \\
\hline $0.2,, \Delta \mathrm{t}=0.001$ & 0.2073 & 0.1162 & 0.0029 & \\
\hline $0.1,, \Delta \mathrm{t}=0.001$ & 0.1670 & 0.0958 & $7.6 \times 10^{-3}$ & \\
\hline $0.05, \Delta \mathrm{t}=0.001$ & 0.1569 & 0.0907 & $1.9 \times 10^{-3}$ & \\
\hline
\end{tabular}




\section{Conclision}

In this paper, a numerical solution of the modified regularized long wave (MRLW) equation has been solved the classical implicit finite-difference technique. In Table, for $\Delta \mathrm{t}=0.001$ value when $L_{2}$ and $L_{\infty}$ error norms are compared with study available in the literature, we have find approximate values. We say that applied method is some good.

\section{References}

Benjamin T.B., Bona, J.L. \& Mahoney, J.L. 1972, Model equations for long waves in nonlinear dispersive media, Philosophical Transactions of the Royal Society A 272, 47-78.

Bona, J.L. \& Pryant, P.J. 1973, A mathematical model for long wave generated by wave makers in nonlinear dispersive systems, Mathematical Proceedings of the Cambridge Philosophical Society, 73: 391-405.

Esen, A. \& Kutluay, S. 2005, Application of lumped Galerkin method to the regularized long wave equation, Applied Mathematics and Computation.

Gardner, L.R.T. \& Gardner, G.A. 1990, Solitary waves of the regularized long wave equation, Journal of Computational Physics, 91: 441-459.

Gou, B.Y. \& Cao, W.M. 1988, The Fourier pseudo-spectral method with a restrain operator for the RLW equation, Journal of Computational Physics, 74: 110-126.

Karakoç BG. Uçar Y. \& Yağmurlu NM.2015, Numerical solutions of the MRLW equation by cubic B-spline Galerkin finite element method, Kuwait J. Sci. 42 (2) pp. 141-159.

Karakoc, S.B.G. \& Geyikli, T. 2013, Petrov-Galerkin finite element method for solving the MRLW equation, Mathematical Sciences, 7:25.

Keskin, P. \& Irk, D.2012, Numerical Solution of the MRLW Equation Using Finite Difference Method International Journal of Nonlinear Science, 14(3), 355-361. 